\title{
Long-Term Outcome of Rectal Cancer With Clinically (EUS/ MRI) Metastatic Mesorectal Lymph Nodes Treated by Neoadjuvant Chemoradiation: Role of Organ Preservation Strategies in Relation to Pathologic Response
}

\author{
Claudio Belluco, MD, $\mathrm{PhD}^{\mathbf{1}}$, Marco Forlin, $\mathrm{MD}^{1}$, Matteo Olivieri, $\mathrm{MD}^{\mathbf{1}}$, Renato Cannizzaro, $\mathrm{MD}^{2}$, \\ Vincenzo Canzonieri, $\mathrm{MD}^{3}$, Angela Buonadonna, $\mathrm{MD}^{4}$, Ettore Bidoli, $\mathrm{ScD}^{5}$, Fabio Matrone, $\mathrm{MD}^{6}$, Giulio Bertola, \\ $\mathrm{MD}^{1}$, and Antonino De Paoli, MD ${ }^{6}$ \\ ${ }^{1}$ Department of Surgical Oncology, CRO-IRCCS, National Cancer Institute, Aviano, Italy; ${ }^{2}$ Department of \\ Gastroenterology, CRO-IRCCS, National Cancer Institute, Aviano, Italy; ${ }^{3}$ Department of Pathology, CRO-IRCCS, \\ National Cancer Institute, Aviano, Italy; ${ }^{4}$ Department of Medical Oncology, CRO-IRCCS, National Cancer Institute, \\ Aviano, Italy; ${ }^{5}$ Department of Epidemiology, CRO-IRCCS, National Cancer Institute, Aviano, Italy; ${ }^{6}$ Department of \\ Radiation Oncology, CRO-IRCCS, National Cancer Institute, Aviano, Italy
}

\begin{abstract}
Background. Organ preservation strategies are under investigation for patients with locally advanced rectal cancer (LARC) who achieve a complete pathologic response in the primary tumor (ypT0) after neoadjuvant chemoradiation therapy (CRT). This study explored the value of this approach for $\mathrm{cN}+$ patients.

Methods. Data were retrieved from our institutional prospective rectal cancer database. Tumors with mesorectal lymph nodes larger than $5 \mathrm{~mm}$ shown on endorectal ultrasonography, pelvic magnetic resonance imaging, or both were staged as $\mathrm{cN}+$.

Results. The study population comprised 226 patients (142 men and 84 women; median age, 64 years) with LARC who underwent CRT followed by surgery including total mesorectal excision (TME) $(n=179)$ and fullthickness local excision (LE) $(n=47)$ between 1996 and 2013. At staging, 123 patients $(54.4 \%)$ were $\mathrm{cN}+$. In 65 cases $(28.7 \%)$, ypCR was observed. Metastatic mesorectal lymph nodes (ypN+) were detected in $41.6 \%$ of the $\mathrm{cN}+$ patients and in $2.8 \%$ of the cN0 patients $(P<0.01)$. Among the $\mathrm{cN}+$ patients, $16 \%$ of the ypT0 cases were
\end{abstract}

(C) The Author(s) 2016. This article is published with open access at Springerlink.com

First Received: 1 April 2016;

Published Online: 3 August 2016

C. Belluco, MD, PhD

e-mail: cbelluco@cro.it
ypN+ compared with $51.8 \%$ of the no-ypT0 cases $(P<0.01)$. Among the $\mathrm{cN}+$ patients who underwent TME, the 5-year disease-specific survival (DSS) and disease-free survival (DFS) rates were respectively 100 and $91.6 \%$ for the ypT0 patients compared with 71.2 and $58.0 \%$ for the no-ypT0 patients $(P=0.01)$. Among the ypN+ patients, the 5-year DSS and DFS rates were both $100 \%$ for the ypT0 cases compared with 59.1 and $43.3 \%$ for the noypT0 patients. Among the $\mathrm{cN}+$ and ypT0 patients, the 5-year DSS and DFS were respectively 100 and $85.7 \%$ for the TME patients compared with 100 and $91.6 \%$ for the LE patients. In the multivariate analysis, ypT0 was the only independent prognostic factor.

Conclusions. Protocols aimed at organ preservation in LARC that achieve ypT0 after CRT can be offered also to $\mathrm{cN}+$ patients.

Neoadjuvant chemoradiation therapy (CRT) and radical surgery including total mesorectal excision (TME) reduces the risk of local recurrence and is considered the standard of care for patients with locally advanced (T3-4 or any N1-2) mid-distal rectal cancer (LARC) ${ }^{1-4}$ A pathologic complete response (ypCR) shown in the surgical specimen of LARC patients treated by CRT is observed in up to one-third of the cases. ${ }^{5}$

In ypCR cases, a favorable long-term oncologic outcome has been observed, ${ }^{6-9}$ and organ preservation strategies including transanal full-thickness local excision and/or close observation are being explored in patients 
displaying clinical or pathologic complete response to CRT. This would lead to a reduction in surgery-related morbidity and mortality and to quality-of-life improvement. $^{10-23}$

The potential presence of metastatic mesorectal lymph nodes, with the related risk of local and distant recurrences, represents a key limiting factor for the application of organ preservation strategies. The reported rate of metastatic mesorectal lymph nodes in the surgical specimen of LARC patients achieving a complete pathologic response (ypT0) in the primary tumor is variable, ${ }^{24-33}$ and the accuracy of lymph node status restaging after CRT is low. ${ }^{34-36}$

Because a priori knowledge of pathologic and oncologic outcome risks is an important issue for protocol design and for clinician-patient communication at clinical study enrollment, we specifically focused this study on patients with rectal cancer staged by endorectal ultrasonography (EUS), pelvic magnetic resonance imaging (MRI), or both as having metastatic mesorectal lymph nodes at their initial diagnosis $(\mathrm{cN}+)$.

To evaluate whether $\mathrm{cN}+$ patients could be reasonably eligible for treatment strategies aimed at organ preservation, we analyzed the pathologic and long-term oncologic outcomes for LARC patients treated by neoadjuvant CRT at our institution during a 17-year period.

\section{METHODS}

All consecutive informed-consent patients treated by neoadjuvant CRT and surgery for LARC between January 1996 and October 2013 were identified from our institutional, prospectively maintained, rectal cancer database. Patients with synchronous distant metastasis were excluded from the study.

All the patients had biopsy-proven adenocarcinoma of the rectum. The distance of the tumor from the anal verge was measured by rigid rectoscopy. Pre- and post-CRT primary tumor and nodal stagings were evaluated by EUS, pelvic MRI, or both. Lymph nodes $5 \mathrm{~mm}$ or larger were considered positive. In cases with discrepancy between the two imaging techniques, the higher stage was considered. Distant metastases were ruled out by thoracoabdominal and pelvic CT scan.
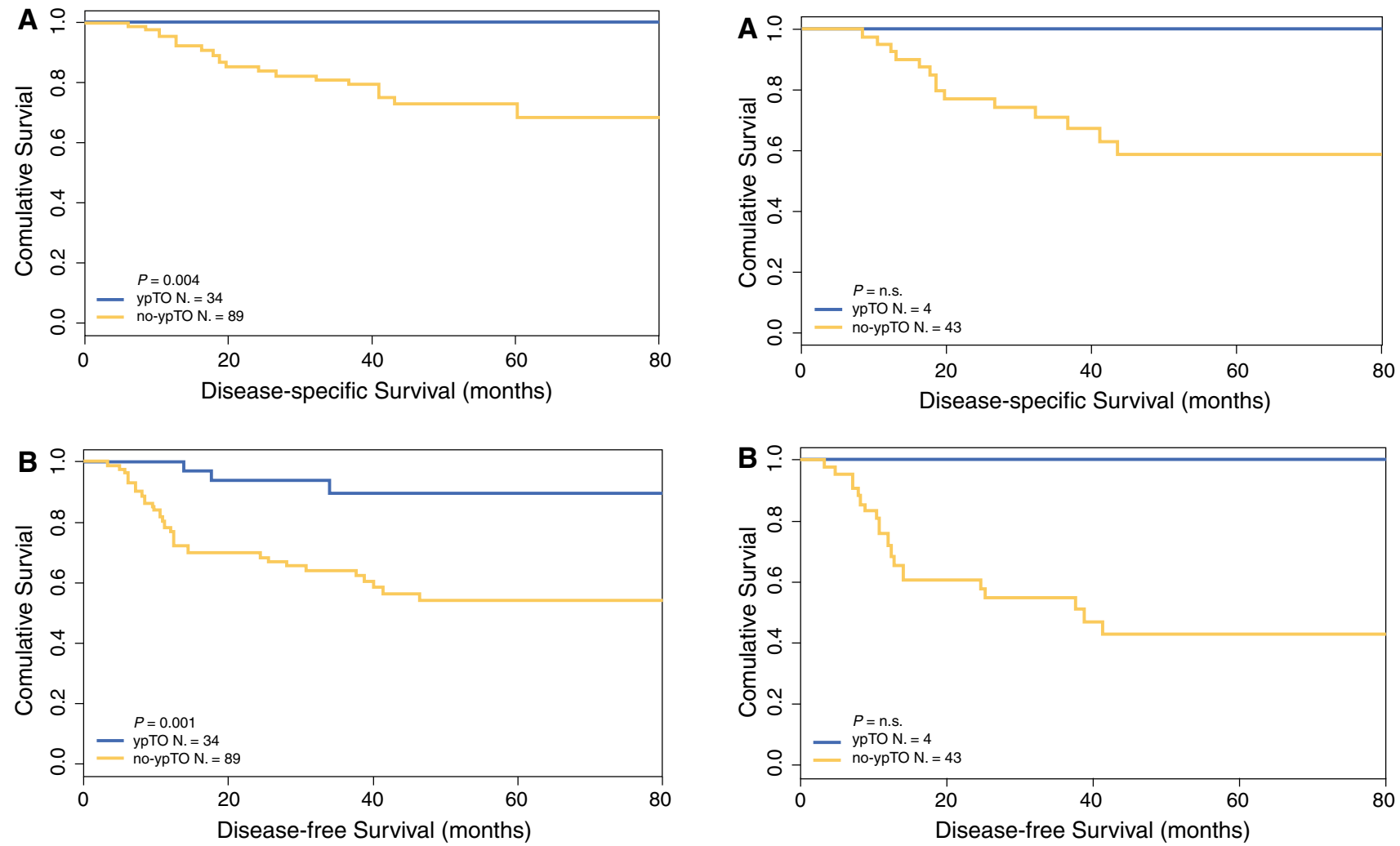

FIG. 1 Kaplan-Meier estimates for disease-specific survival (a) and disease-free survival (b) according to a complete pathologic response of the primary tumor (ypT0) in $123 \mathrm{cN}+$ rectal cancer patients treated by neoadjuvant chemoradiation followed by total mesorectal excision (TME) or full-thickness local excision (LE) surgery

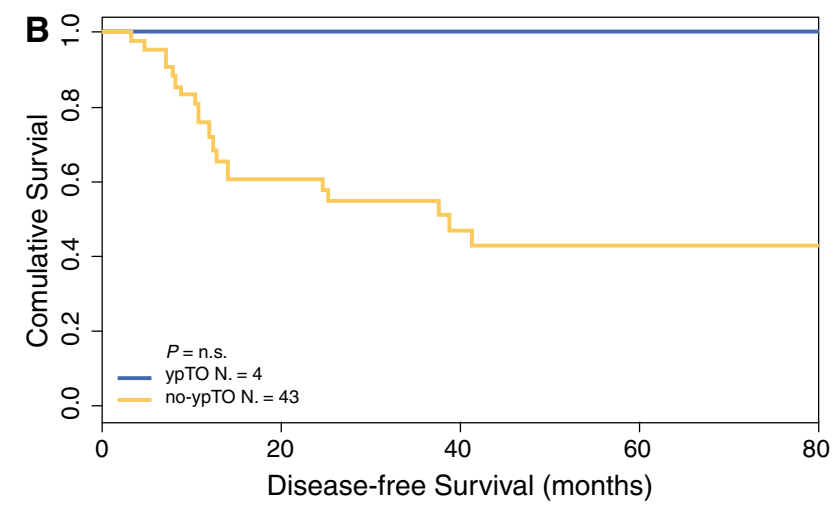

FIG. 2 Kaplan-Meier estimates for disease-specific survival (a) and disease-free survival (b) according to a complete pathologic response of the primary tumor (ypT0) in $47 \mathrm{ypN}+$ rectal cancer patients treated by neoadjuvant chemoradiation followed by total mesorectal excision (TME) surgery 
TABLE 1 Long-term oncologic outcome according to clinocopathologic characteristics in locally advanced rectal cancer patients treated by neoadjuvant chemoradiation

\begin{tabular}{|c|c|c|c|c|c|c|c|}
\hline \multirow[t]{2}{*}{ Variable } & \multirow{2}{*}{$\begin{array}{l}\text { Total } \\
n(\%)\end{array}$} & \multicolumn{2}{|c|}{ 5-yrs DSS } & \multicolumn{2}{|c|}{ 5-yrs DFS } & \multicolumn{2}{|c|}{ 5-yrs LRFS } \\
\hline & & $\%$ & $P$ Value & $\%$ & $P$ Value & $\%$ & $P$ Value \\
\hline \multicolumn{8}{|l|}{ Sex } \\
\hline Female & $84(37.2)$ & 80.2 & 0.638 & 69.9 & 0.461 & 89.7 & 0.042 \\
\hline Male & $142(62.8)$ & 84.4 & & 65.1 & & 78.0 & \\
\hline \multicolumn{8}{|l|}{ Age (years) } \\
\hline$\leq 65$ & $128(56.6)$ & 82.2 & 0.863 & 67.5 & 0.896 & 82.8 & 0.874 \\
\hline$>65$ & $98(43.4)$ & 84.0 & & 65.9 & & 82.1 & \\
\hline \multicolumn{8}{|l|}{$\mathrm{cN}$ status } \\
\hline $\mathrm{cNO}$ & 103 (45.6) & 86.3 & 0.537 & 69.7 & 0.537 & 83.0 & 0.872 \\
\hline $\mathrm{cN}+$ & $123(54.4)$ & 80.5 & & 64.2 & & 82.2 & \\
\hline \multicolumn{8}{|c|}{ Type of surgery } \\
\hline TME & $179(79.2)$ & 81.8 & 0.201 & 66.4 & 0.700 & 84.0 & 0.312 \\
\hline LE & $47(20.8)$ & 87.2 & & 68.2 & & 76.8 & \\
\hline \multicolumn{8}{|l|}{ ypT status } \\
\hline урТ0 & $65(28.7)$ & 94.5 & 0.005 & 87.3 & $<0.001$ & 90.5 & 0.034 \\
\hline No ypT0 & $161(71.3)$ & 78.4 & & 58.8 & & 79.3 & \\
\hline ypN status & & & & 66.2 & & 80.9 & \\
\hline ypNO & $132(73.8)$ & 88.0 & 0.002 & 72.2 & 0.008 & 85.4 & 0.309 \\
\hline ypN+ & $47(26.2)$ & 71.7 & & 55.7 & & 85.7 & \\
\hline
\end{tabular}

$D S S$ disease-specific survival, DFS disease-free survival, $L R F S$ local-recurrence-free survival, $c N$ clinical lymph node, $T M E$ total mesorectal excision, $L E$ local excision, $y p T 0$ complete pathologic response in the primary tumor, $y p N$ pathologic lymph node status (only TME patients)

TABLE 2 Metastatic lymph node rates in locally advanced rectal cancer with complete pathologic response in the primary tumor (ypT0) achieved after neoadjuvant chemoradiation

\begin{tabular}{|c|c|c|c|c|c|c|}
\hline & \multirow[t]{2}{*}{ No. of patients } & \multirow[t]{2}{*}{ Examined lymph nodes median (range) } & \multicolumn{2}{|c|}{ YpT0 } & \multicolumn{2}{|c|}{$\mathrm{YpN}+$} \\
\hline & & & $N$ & $\%$ & $N$ & $\%$ \\
\hline Read et al. ${ }^{24}$ & 644 & $13 \pm 8$ & 42 & 6.52 & 1 & 2.38 \\
\hline Pucciarelli et al. ${ }^{25}$ & 235 & $9(0-38)$ & 56 & 23.83 & 1 & 1.79 \\
\hline Hughes et al. ${ }^{26}$ & 130 & $6(0-21)$ & 23 & 17.69 & 4 & 17.39 \\
\hline Guillem and Minsky $^{27}$ & 188 & $9(0-38)$ & 37 & 19.68 & 1 & 2.70 \\
\hline Berho et al. ${ }^{28}$ & 86 & $13,1(1-59)$ & 18 & 20.93 & 2 & 11.11 \\
\hline Yeo et al. ${ }^{29}$ & 333 (all ypT0) & $10(0-78)$ & 333 & 100 & 29 & 8.70 \\
\hline Jang et al. ${ }^{30}$ & 830 & 11 & 91 & 10.96 & 6 & 6.59 \\
\hline Tranchart et al. ${ }^{31}$ & 245 & $24(3-60)$ & 26 & 10.61 & 2 & 7.69 \\
\hline Park et al. ${ }^{32}$ & 725 & $11(6-15)$ & 143 & 19.72 & 13 & 9.09 \\
\hline Sprenger et al. ${ }^{33}$ & 398 & $28.0 \pm 13.7$ & 40 & 10.0 & 4 & 10.00 \\
\hline Current study & 179 & $13(2-37)$ & 40 & 22.34 & 4 & 10.00 \\
\hline
\end{tabular}

$y p T 0$ complete pathologic response in the primary tumor, $y p N$ pathologic lymph node status

\section{Treatment}

Preoperative CRT Preoperative CRT was administered according to several preoperative sequential treatment protocols developed at our institution, including a 5-fluorouracil (5-FU) bolus + leucovorin (LV) and 45 Gy with or withut adjuvant 5-FU/LV, raltitrexed and $50.4+10$ Gy of intraoperative radiation therapy (IORT), capecitabine and $50.4 \mathrm{~Gy}$, continuous infusion 5 -FU + gefitinib and $50.4+10 \mathrm{~Gy}$ IORT, and 
capecitabine \pm oxaliplatin and 50.4 Gy. The radiotherapy (RT) clinical target volume (CTV2) included the primary tumor, the mesorectum, and internal iliac lymph nodes. A second clinical target volume (CTV1) included the mesorectum corresponding to the primary tumor with a 2-cm radial margin. The RT fractionation was 180 cGy/day, 5 fractions per week. More details on RT technique and dose prescription have been reported previously. ${ }^{8}$

Surgery The patients underwent surgery 6-8 weeks after completion of neoadjuvant CRT. The surgical procedures included abdominoperineal resection (APR), low anterior resection (LAR), and full-thickness transanal local excision (LE). Radical resection was performed according to TME principles. Reasons for the use of LE included medical comorbidity and patient refusal of APR for low-lying tumors not eligible for coloanal reconstruction due to anticipation of poor sphincter function.

In more recent years, patients with a major clinical response to CRT were offered the option of LE in a prospective clinical study investigating the outcome of LE after a complete clinical and pathologic response. In these cases, LE was used to assess the pathologic response in the primary tumor. Medically fit patients showing no complete or almost complete pathologic response in the primary tumor (TRG1 and TRG2 according to Mandard tumor response grading $)^{37}$ underwent subsequent TME surgery. After surgical resection, IORT to a high risk area (presacral region) was administered according to study protocols, as mentioned earlier.

\section{Postoperative Chemotherapy Adjuvant 5-FU-based} chemotherapy was administered according to the study protocol, or in selected cases included patients with metastatic lymph nodes.

\section{Pathology}

Pathologic tumor staging was performed according to the guidelines of the American Joint Committee on Cancer and the College of American Pathologists. ${ }^{38}$ Patients with no residual cancer cells in the surgical specimen were considered pathologic complete responders (ypCR).

\section{Follow-up Evaluation}

Postoperatively, the patients were examined at followup visits every 3 months for the first 2 years and halfyearly thereafter. At each follow-up control visit, the CEA level was determined. Abdominal and pelvic computed tomography (CT) scan or liver ultrasound and chest x-ray were performed alternatively every 3-6 months. Colonoscopy was performed yearly.

\section{Statistical Analysis}

The Chi square test or Fisher's exact test was used to compare percentages between complete responders and non-complete responders, and the Wilcoxon rank test was used for median age comparison. Cumulative probabilities of overall survival (OS), disease-specific survival (DSS), disease-free survival (DFS), distant metastasis-free survival (DMFS), and local recurrence-free survival (LRFS) were estimated by Kaplan-Meier survival methods, ${ }^{39}$ and differences between subgroups were assessed using the logrank test. The duration of follow-up evaluation was calculated as the time from surgery to the event of interest. Patients without event were censored at the date of the last follow-up visit. In cases with local and distant metastasis, both events were recorded and computed at any time of occurrence. For better assessment of the oncologic implications of ypCR, the Cox proportional hazards model was used to adjust the hazard ratios (HRs) and corresponding $95 \%$ confidence intervals (CIs).$^{40}$ Due to the limitation of sample size and number of events, only three variables were entered into the multivariate model: cNstage ( $\mathrm{cNO}$ vs cN1), type of surgery (TME vs LE), and ypT0 (yes vs no). Collinearity between variables was excluded by means of the Chi square test. A $P$ value of 0.05 or lower was considered statistically significant (two-tailed). The SAS System 9.2 (SAS, Cary, NC, USA) was used as the statistical software for data analysis.

\section{RESULTS}

\section{Patients and Treatment Characteristics}

The study population comprised 226 consecutive patients (142 men and 84 women; median age, 64 years; range, 25-87 years) with mid-distal LARC and no distant metastasis treated by neoadjuvant CRT followed by surgery at our institution between January 1996 and October 2013.

At the initial evaluation, 226 patients were staged as follows: 5 cT2N1 (2.2 \%), 79 cT3N0 (34.9\%), 104 cT3N1 (46 \%), 12 cT4N0 (5.3\%), 13 cT4N1 (5.7\%), 2 cTxN0 $(0.8 \%)$, and $1 \mathrm{cTxN} 1(0.4 \%)$. In addition, 10 very lowlying cT2N0 tumors $(4.4 \%)$ were considered at high risk for recurrence, treated by neoadjuvant CRT, and included in this study. The median distance of the tumor from the anal verge was $5 \mathrm{~cm}$ (range, $1-12 \mathrm{~cm}$ ). The total RT dose was 45 Gy for 42 patients (18.5\%), 50.4 Gy for 180 patients $(79.6 \%)$, and 25 Gy for 4 patients $(1.7 \%)$. Total 
mesorectal excision was performed for 179 patients (79 \%) (142 LAR and 37 APR), whereas LE was performed for 47 patients $(21 \%)$. The documented reasons for the use of LE were preference after a major clinical response in 22 cases, patient absolute refusal of APR in 4 cases, and medical comorbidity in 3 cases. The remaining 18 patients were enrolled in the prospective clinical study investigating the outcome for LE after complete clinical and pathologic response. All patients restaged as $\mathrm{ycN}+(n=24)$ underwent TME surgery. Intraoperative radiation therapy was applied in the context of clinical studies. Postoperative chemotherapy was administered to all $33 \mathrm{ypN}+$ patients $(14 \%)$.

\section{Clinical and Pathologic Response}

In the entire patient population, a complete pathologic response in the primary tumor (ypT0) was observed in 65 cases $(28.7 \%)$. For the 179 patients who underwent TME, the ypCR rate (ypT0N0) was $20.1 \%(n=36)$. The median number of examined lymph nodes was 13 (range, 2-37). Metastatic lymph nodes (ypN+) were found in $47(26.2 \%)$ of the surgical specimens: in $4(10 \%)$ of $40 \mathrm{ypT} 0$ cases, in $1(9 \%)$ of 11 ypT1 cases, in 12 (21\%) of 57 ypT2 cases, in $28(43 \%)$ of 65 ypT3 cases, and in $2(33.3 \%)$ of 6 ypT4 cases.

Among the patients who underwent TME surgery, metastatic lymph nodes (ypN+) were detected in 45 $(41.6 \%)$ of $108 \mathrm{cN}+$ patients compared with $2(2.8 \%)$ of $71 \mathrm{cN} 0$ patients $(P<0.01)$.

In the subgroup of $\mathrm{cN}+$ tumors with ypT0 treated by TME surgery, 4 (16\%) of 25 (all restaged as ycN0) were ypN+ compared with $43(51.8 \%)$ of 83 cases that had no ypT0 $(P<0.01)$.

In the subgroup of $\mathrm{cN} 0$ tumors with ypT0 treated by TME surgery, 0 of 15 were ypN+ compared with $2(3.5 \%)$ of 56 no-ypT0 cases.

At restaging after CRT, comparing ycN status with ypN status, metastatic lymph nodes at pathology were detected in 9 of $23 \mathrm{ycN}+$ cases and in 10 of $25 \mathrm{ycN} 0$ cases (sensitivity, 0.47; specificity, 0.51).

\section{Recurrence and Survival}

No postoperative mortality occurred. During a median follow-up period of 48 months, 20 patients (8.84\%) experienced local recurrence only, $14(6.19 \%)$ experienced local recurrence and distant metastasis (9 liver, 4 lung and 1 other site cases), and 34 (15.04\%) experienced distant metastasis only (15 liver, 9 lung, 7 liver and lung, and 3 multiple-site cases).

In the comparison of ypCR-patients (ypTON0) and noypCR patients who underwent TME surgery, the 5-year
DSS was respectively 91.0 and $79.4 \%(P=0.029)$, and the 5-year DFS was 84.9 and $61.7 \%(P=0.011)$.

In the entire patient population, the 5-year survival rates were $79.2 \%$ for OS, $83.0 \%$ for DSS, $66.9 \%$ for DFS, $77.1 \%$ for DMFS, and $82.6 \%$ for LRFS. In the subset of 65 ypT0 patients, 2 (3.1\%) experienced local recurrence only, $3(4.61 \%)$ experienced local recurrence and distant metastasis, and $3(4.61 \%)$ experienced distant metastasis only (1 liver, and 2 liver and lung cases).

In the comparison of ypT0 patients $(n=65)$ with noypT0 patients $(n=161)$, the 5 -year OS was respectively 89.4 versus $75.3 \%(P=0.005)$, the 5-year DSS was 94.5 versus $78.4 \%(P=0.005)$, the 5-year DFS was 87.3 versus $58.8 \%(P<0.001)$, the 5-year DMFS was 93.0 versus $70.6 \%(P=0.002)$, and the 5-year LRFS was 90.5 versus $79.3 \%(P=0.034)$. According to the clinical lymph node status at initial diagnosis, the 5-year DSS and DFS were respectively 80.5 and $64.2 \%$ in $\mathrm{cN}+$ cases compared with 86.3 and $69.7 \%$ in cN0 cases (nonsignificant difference) (Table 1).

Among the $\mathrm{cN}+$ patients $(n=123)$ the 5 -year DSS and DFS were respectively 100 and $91.6 \%$ for the ypT0 patients compared with 71.2 and $58.0 \%$ for the no-ypT0 patients $(P<0.01$; Fig. 1$)$. The 5 -year DSS and DFS were both $100 \%$ for the 4 ypT0N+ patients compared with 59.1 and $43.3 \%$ respectively for the 43 no-ypTN+ patients (nonsignificant difference; Fig. 2).

Among the $\mathrm{cN}+$ patients who achieved ypT0, the 5-year DSS and DFS were respectively 100 and $85.7 \%$ for the TME patients $(n=108)$ compared with 100 and $91.6 \%$ for the LE patients ( $n=15$ ) (nonsignificant difference). In the multivariate analysis, ypT0 was the only independent prognostic factor for DSS (HR, 0.13; $95 \%$ CI, 0.03-0.58; $P=0.007)$ and for DFS (HR, 0.25; $95 \%$ CI, 0.12-0.54; $P<0.001)$.

\section{DISCUSSION}

The current study investigated whether LARC patients initially staged as $\mathrm{cN}+$ and achieving ypT0 after neoadjuvant CRT are potential candidates for organ-preserving surgical strategies. To this end, the rate of ypT0, the incidence of metastatic lymph nodes, and the long-term oncologic outcome were analyzed in relation to $\mathrm{cN}$ status in LARC patients treated by neoadjuvant CRT and prospectively followed up at a single institution.

For our patients treated with TME surgery after CRT, ypCR was achieved in $20.1 \%$ of the cases, which is in line with the majority of studies previously reported in the literature. ${ }^{5}$ Our survival analysis supported the evidence of a favorable long-term oncologic outcome for patients displaying ypCR. In our series comparing ypCR patients with 
no-ypCR patients, the 5-year DFS rates were respectively 84.9 and $61.7 \%$. This is in line with the data reported by Maas et al. ${ }^{9}$ from a pooled analysis of 3105 LARC patients treated by preoperative CRT who showed a 5-year DFS of $83.3 \%$ for ypCR patients compared with $65.6 \%$ for noypCR patients. Similarly in a meta-analysis by Zorcolo et al. $^{41}$ of 12 studies including 1913 LARC patients, the 5 -year DFS was $86.9 \%$ for ypCR patients compared with $63.9 \%$ for no-ypCR patients. Recently Wasmooth et al. ${ }^{42}$ reported a 5-year DFS of $81 \%$ for patients with ypCR and $50 \%$ for patients without ypCR among 1384 patients enrolled in the national population-based colorectal cancer registry of Norway who had advanced $\mathrm{T} 3$ and $\mathrm{T} 4$ rectal cancer with N0-2,M0 managed by neoadjuvant long-course (chemo)radiation. Interestingly, ypCR was associated with a low risk of metastasizing.

In our subset of ypT0 patients treated by LE surgery, the local and distant recurrence rates were very low and similar to those for ypT0 patients treated by TME surgery. This finding is consistent with data reported by Borshitz et al., ${ }^{13}$ who analyzed seven studies reporting oncologic outcome of LE after neoadjuvant CRT for cT2-3 tumors $(n=237)$. In their study, ypT0 was noted in $22 \%$ of the cases, and the 5-year LRFS and DMFS were respectively 100 and $96 \%$. Similarly, Pucciarelli et al. ${ }^{18}$ reported that the 3-year LRFS was $96.9 \%$ for 43 cT3 or low-lying cT2 rectal cancer patients treated with CRT followed by LE and observation for the ypT0-1 patients.

In our patients initially staged as $\mathrm{cN}+$ and treated with CRT followed by TME surgery, metastatic lymph nodes at pathology were detected in $42.2 \%$ of the cases. However, in the subgroup of patients with ypT0, metastatic lymph nodes were detected in $16 \%$ of the surgical specimens. The rate of metastatic lymph nodes in LARC achieving ypT0 after CRT has been reported to vary between 2 and $17 \%$, which is in line with our findings of a $10 \%$ rate (Table 2). ${ }^{24-33}$

The assessment of response to treatment is becoming increasingly important in view of a personalized surgical approach. Among our patients, restaging accuracy using standard MRI and endorectal ultrasound was very low. This is in line with two recent meta-analysis leading to the conclusion that overall accuracy of restaging is not sufficiently consistent for clinical application. ${ }^{34,35}$ In addition, a nomogram using clinicopathologic parameters to predict ypN status after CRT developed in a training cohort of 891 LARC patients has been shown to achieve an accuracy of 0.77 in an external validation cohort of 258 patients. ${ }^{36}$

In view of the aforementioned limitations, even if surgical complications, including suture dehiscence and endoanal pain, are not uncommon among patients undergoing LE after CRT, as previously reported by us and others, ${ }^{18,43}$ this remains a procedure of investigational interest to confirm potential ypT0 status of patients with a major clinical response. On the other hand, a more conservative approach such as the "wait and see" option might be considered for patients with a complete clinical response. ${ }^{44}$ Hopefully, new techniques such as the fluorodeoxyglucose (FDG)-positron emission tomography (PET) scan and perfusion MRI might lead to a precise assessment of response. ${ }^{45-48}$

This study was limited by its single-center retrospective design and its small number of ypT0 patients displaying metastatic lymph nodes at pathology. In addition, the large time frame considered might have accounted for our relatively high local recurrence rate compared with the results of prospective clinical studies.

In conclusion, our findings indicate that treatment protocols aimed at organ preservation in rectal cancer achieving ypT0 after CRT can be offered also to patients with clinically positive mesorectal lymph nodes at their initial diagnosis. The favorable long-term outcome for ypT0 tumors and the risk of metastatic mesorectal lymph nodes should be discussed in patient-clinician communication.

DISCLOSURE All protocols were approved by our institutional review committee and all patients were informed consent.

OPEN ACCESS This article is distributed under the terms of the Creative Commons Attribution 4.0 International License (http:// creativecommons.org/licenses/by/4.0/), which permits unrestricted use, distribution, and reproduction in any medium, provided you give appropriate credit to the original author(s) and the source, provide a link to the Creative Commons license, and indicate if changes were made.

\section{REFERENCES}

1. Sauer R, Becker H, Hohenberger W, et al. Preoperative versus postoperative chemoradiotherapy for rectal cancer. $N$ Engl J Med. 2004;351:1731-40.

2. Bosset J-F, Collette L, Calais G, et al. Chemotherapy with preoperative radiotherapy in rectal cancer. $N$ Engl $J$ Med. 2006;355:1114-23.

3. Rahbari NN, Elbers H, Askoxylakis V, et al. Neoadjuvant radiotherapy for rectal cancer: meta-analysis of randomized controlled trials. Ann Surg Oncol. 2013;20:4169-82. doi:10.1245/ s10434-013-3198-9.

4. NCCN Clinical Practice Guidelines in Oncology. Rectal cancer (version 1.2016). http://www.nccn.org/professionals/physician_ gls/pdf/rectal.pdf. Accessed 23 Feb 2016.

5. Sanghera P, Wong DW, McConkey CC, Geh JI, Hartley A. Chemoradiotherapy for rectal cancer: an updated analysis of factors affecting pathological response. Clin Oncol R Coll Radiol. 2008;20:176-83. doi:10.1016/j.clon.2007.11.013.

6. Fokas E, Liersch T, Fietkau R, et al. Tumor regression grading after preoperative chemoradiotherapy for locally advanced rectal carcinoma revisited: updated results of the CAO/ARO/AIO-94 trial. J Clin Oncol. 2014;32:1554-62. doi:10.1200/JCO.2013.54. 3769. 
7. Park IJ, You YN, Agarwal A, et al. Neoadjuvant treatment response as an early response indicator for patients with rectal cancer. J Clin Oncol. 2012;30:1770-6. doi:10.1200/JCO.2011.39. 7901.

8. Belluco C, De Paoli A, Canzonieri V, et al. Long-term outcome of patients with complete pathological response after neoadjuvant chemoradiation for cT3 rectal cancer: implications for local excision surgical strategies. Ann Surg Oncol. 2011;18:3686-93. doi:10.1245/s10434-011-1822-0.

9. Maas M, Nelemans PJ, Valentini V, et al. Long-term outcome in patients with a pathological complete response after chemoradiation for rectal cancer: a pooled analysis of individual patient data. Lancet Oncol. 2010;11:835-44.

10. Callender GG, Das P, Rodriguez-Bigas MA, et al. Local excision after preoperative chemoradiation results in an equivalent outcome to total mesorectal excision in selected patients with T3 rectal cancer. Ann Surg Oncol. 2010;17:441-7. doi:10.1245/ s10434-009-0735-7.

11. Guerrieri M, Baldarelli M, Organetti L, Grillo Ruggeri F, Mantello G, Bartolacci S, et al. Transanal endoscopic microsurgery for the treatment of selected patients with distal rectal cancer: 15 years experience. Surg Endosc. 2008;22:2030-5. doi:10.1007/ s00464-008-9976-y.

12. Nair RM, Siegel EM, Chen DT, et al. Long-term results of transanal excision after neoadjuvant chemoradiation for $\mathrm{T} 2$ and T3 adenocarcinomas of the rectum. $J$ Gastrointest Surg. 2008;12:1797-805. doi:10.1007/s11605-008-0647-z.

13. Borschitz T, Wachtlin D, Möhler M, Schmidberger H, Junginger T. Neoadjuvant chemoradiation and local excision for T2-3 rectal cancer. Ann Surg Oncol. 2008;15:712-20. doi:10.1245/s10434007-9732-x.

14. Bonnen M, Crane C, Vauthey JN, et al. Long-term results using local excision after preoperative chemoradiation among selected T3 rectal cancer patients. Int $J$ Radiat Oncol Biol Phys. 2004;60:1098-105. doi:10.1016/j.ijrobp.2004.07.368.

15. Ruo L, Guillem JG, Minsky BD, Quan SH, Paty PB, Cohen AM. Preoperative radiation with or without chemotherapy and fullthickness transanal excision for selected $\mathrm{T} 2$ and $\mathrm{T} 3$ distal rectal cancers. Int J Colorectal Dis. 2002;17:54-8. doi:10.1007/ s003840100327.

16. Kim CJ, Yeatman TJ, Coppola D, et al. Local excision of T2 and T3 rectal cancers after downstaging chemoradiation. Ann Surg. 2001;234:352-8. doi:10.1097/00000658-200109000-00009.

17. Schell SR, Zlotecki RA, Mendenhall WM, Marsh RW, Vauthey JN, Copeland EM III. Transanal excision of locally advanced rectal cancers downstaged using neoadjuvant chemoradiotherapy. $J$ Am Coll Surg. 2002;194:584-90. doi:10.1016/S10727515(02)01128-6.

18. Pucciarelli S, De Paoli A, Guerrieri M, et al. Local excision after preoperative chemoradiotherapy for rectal cancer: results of a multicenter phase II clinical trial. Dis Colon Rectum. 2013;56:1349-56.

19. Habr-Gama A, Gama-Rodrigues J, Sao Juliao GP, et al. Local recurrence after complete clinical response and watch and wait in rectal cancer after neoadjuvant chemoradiation: impact of salvage therapy on local disease control. Int J Radiat Oncol Biol Phys. 2014;88:822-8.

20. Maas M, Beets-Tan RG, Lambregts DM, et al. Wait-and-see policy for clinical complete responders after chemoradiation for rectal cancer. J Clin Oncol. 2011;29:4633-40.

21. Appelt AL, Ploen J, Harling H, et al. High-dose chemoradiotherapy and watchful waiting for distal rectal cancer: a prospective observational study. Lancet Oncol. 2015;16:919-27.

22. Smith JD, Ruby JA, Goodman KA, et al. Nonoperative management of rectal cancer with complete clinical response after neoadjuvant therapy. Ann Surg. 2012;256:965-72.
23. Smith RK, Fry RD, Mahmoud NN, Paulson EC. Surveillance after neoadjuvant therapy in advanced rectal cancer with complete clinical response can have comparable outcomes to total mesorectal excision. Int J Colorectal Dis. 2015;30:769-74.

24. Read TE, Andujar JE, Caushaj PF, et al. Neoadjuvant therapy for rectal cancer: histologic response of the primary tumor predicts nodal status. Dis Colon Rectum. 2004;47:825-31.

25. Pucciarelli S, Capirci C, Emanuele U, et al. Relationship between pathological $\mathrm{T}$-stage and nodal metastasis after preoperative chemoradiotherapy for locally advanced rectal cancer. Ann Surg Oncol. 2005;12:111-6.

26. Hughes R, Glynne-Jones R, Grainger J, et al. Can pathological complete response in the primary tumour following pre-operative pelvic chemoradiotherapy for T3-T4 rectal cancer predict for sterilisation of pelvic lymph nodes, a low risk of local recurrence and the appropriateness of local excision?. Int J Colorectal Dis. 2006;21:11-7.

27. Guillem JG, Minsky BD. Extended perineal resection of distal rectal cancers: surgical advance, increased utilization of neoadjuvant therapies, proper patient selection or all of the above?. $J$ Clin Oncol. 2008;26:3481-2. doi:10.1200/JCO.2007.15.6646.

28. Berho M, Oviedo M, Stone E, et al. The correlation between tumour regression grade and lymph node status after chemoradiation in rectal cancer. Colorectal Dis. 2009;11:254-8. doi:10. 1111/j.1463-1318.2008.01597.x. Epub 29 May 2008.

29. Yeo SG, Kim DY, Kim TH, et al. Pathological complete response of primary tumor following preoperative chemoradiotherapy for locally advanced rectal cancer: long-term outcomes and prognostic significance of pathological nodal status (KROG 09-01). Ann Surg. 2010;252:998-1004. doi:10.1097/SLA.0b013e3181f3f1b1. PubMed PMID: 21107110.

30. Jang TY, Yu CS, Yoon YS, et al. Oncologic outcome after preoperative chemoradiotherapy in patients with pathological T0 (ypT0) rectal cancer. Dis Colon Rectum. 2012;55:1024-31. doi:10.1097/DCR.0b013e3182644334.

31. Tranchart H, Lefèvre JH, Svrcek M, Flejou JF, Tiret E, Parc Y. What is the incidence of metastatic lymph node involvement after significant pathological response of primary tumor following neoadjuvant treatment for locally advanced rectal cancer?. Ann Surg Oncol. 2013;20:1551-9. doi:10.1245/s10434-012-2773-9.

32. Park IJ, You YN, Skibber JM, Rodriguez-Bigas MA, Feig B, Nguyen $\mathrm{S}$, et al. Comparative analysis of lymph node metastases in patients with ypT0-2 rectal cancers after neoadjuvant chemoradiotherapy. Dis Colon Rectum. 2013;56:135-41. doi:10. 1097/DCR.0b013e318278ff8a.

33. Sprenger T, Rothe H, Conradi LC, et al. Stage-dependent frequency of lymph node metastases in patients with rectal carcinoma after preoperative chemoradiation: results from the CAO/ARO/AIO-94 Trial and from a comparative prospective evaluation with extensive pathological workup. Dis Colon Rectum. 2016;59:377-85.

34. Zhao YL, Cao DM, Zhou QC, Yang N, Yao HL. Accuracy of endorectal endoscopic ultrasound (EUS) for locally advanced rectal cancer (LARC) restaging after neoadjuvant chemoradiotherapy (NAT): a meta-analysis (review). Hepatogastroenterology. 2014;61:978-83.

35. Memon S, Lynch AC, Bressel M, Wise AG, Heriot AG. Systematic review and meta-analysis of the accuracy of MRI and endorectal ultrasound in the restaging and response assessment of rectal cancer following neoadjuvant therapy. Colorectal Dis. 2015;17:748-61. doi:10.1111/codi.12976.

36. Jwa E, Kim JH, Han S, et al. Nomogram to predict ypN status after chemoradiation in patients with locally advanced rectal cancer. $\mathrm{BrJ}$ Cancer. 2014;111:249-54. doi:10.1038/bjc.2014.256.

37. Mandard AM, Dalibard F, Mandard JC, et al. Pathologic assessment of tumor regression after preoperative chemoradiotherapy of 
esophageal carcinoma: clinicopathologic correlations. Cancer. 1994;73:2680-6.

38. Edge SB, Byrd DR, Compton CC, Fritz AG, Greene FL, Trotti A III. AJCC-Cancer Staging Manual. 7th ed. Springer, New York, 2010.

39. Kaplan EL, Meier P. Nonparametric estimation from incomplete observations. J Am Stat Assoc. 1958;53:457-81.

40. Cox DR. Regression model and life tables. $J$ R Stat Soc $B$. 1972;34:178-220.

41. Zorcolo L, Rosman AS, Restivo A, Pisano M, Nigri GR, Fancellu $\mathrm{A}$, et al. Complete pathological response after combined modality treatment for rectal cancer and long-term survival: a meta-analysis. Ann Surg Oncol. 2012;19:2822-32. doi:10.1245/s10434011-2209-y.

42. Wasmuth HH, Rekstad LC, Tran $\varnothing$ G. The outcome and the frequency of pathological complete response after neoadjuvant radiotherapy in curative resections for advanced rectal cancer: a population-based study. Colorectal Dis. 2016;18:67-72. doi:10. 1111/codi.13072.

43. Garcia-Aguilar J, Renfro LA, Chow OS, et al. Organ preservation for clinical T2N0 distal rectal cancer using neoadjuvant chemoradiotherapy and local excision (ACOSOG Z6041): results of an open-label, single-arm, multi-institutional, phase 2 trial.
Lancet Oncol. 2015;16:1537-46. doi:10.1016/S1470-2045(15) 00215-6.

44. Habr-Gama A. Assessment and management of the complete clinical response of rectal cancer to chemoradiotherapy. Colorectal Dis. 2006;8(Suppl 3):21-4.

45. Lambregts DM, Beets GL, Maas M, et al. Accuracy of gadofosveset-enhanced MRI for nodal staging and restaging in rectal cancer. Ann Surg. 2011;253:539-45. doi:10.1097/SLA. 0b013e31820b01f1.

46. Memon S, Lynch AC, Akhurst T, Ngan SY, Warrier SK, Michael $\mathrm{M}$, et al. Systematic review of FDG-PET prediction of complete pathological response and survival in rectal cancer. Ann Surg Oncol. 2014;21:3598-607. doi:10.1245/s10434-014-3753-z.

47. Martens MH, van Heeswijk MM, van den Broek JJ, et al. Prospective, multicenter validation study of magnetic resonance volumetry for response assessment after preoperative chemoradiation in rectal cancer: can the results in the literature be reproduced? Int J Radiat Oncol Biol Phys. 2015;93:1005-14. doi:10.1016/j.ijrobp.2015.09.008.

48. Schneider DA, Akhurst TJ, Ngan SY, et al. Relative value of restaging MRI, CT, and FDG-PET scan after preoperative chemoradiation for rectal cancer. Dis Colon Rectum. 2016;59:179-86. doi:10.1097/DCR.0000000000000557. 